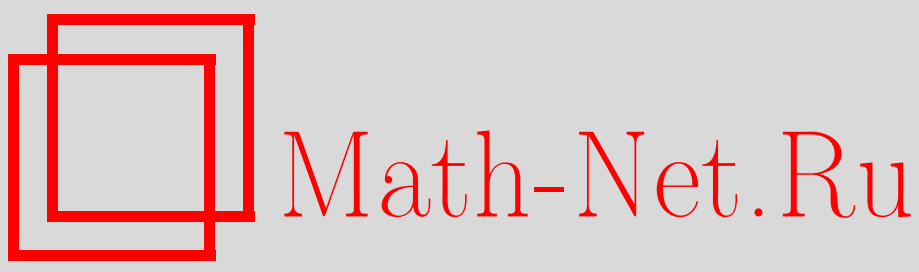

A. G. Pinus, Algebraic functions and inner homomorphisms of universal algebras, Sibirsk. Mat. Zh., 2020, Volume 61, Number 3, 669-673

DOI: https://doi.org/10.33048/smzh.2020.61.314

Use of the all-Russian mathematical portal Math-Net.Ru implies that you have read and agreed to these terms of use http://www . mathnet.ru/eng/agreement

Download details:

IP : 54.197 .217 .227

April 26, 2023, 15:44:26

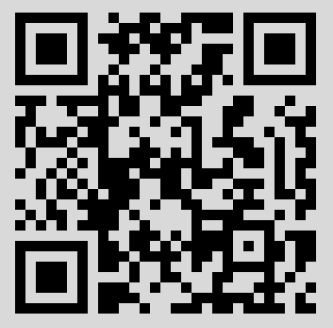


Сибирский математический журнал Май-июнь, 2020. Том 61, № 3

УДК 512.56

\title{
АЛГЕБРАИЧЕСКИЕ ФУНКЦИИ И ВНУТРЕННИЕ ГОМОМОРФИЗМЫ УНИВЕРСАЛЬНЫХ АЛГЕБР
}

\section{А. Г. Пинус}

\begin{abstract}
Аннотация. На основе понятия алгебраической функции в теории функций действительного переменного исследуется вопрос о синтаксическом задании на универсальных алгебрах функций, графики которых являются алгебраическими или кусочно алгебраическими множествами.
\end{abstract}

DOI 10.33048/smzh.2020.61.314

Ключевые слова: алгебраические, глобально алгебраические и квазинеявные функции, внутренние гомоморфизмы универсальных алгебр.

Юрию Леонидовичу Ершову, моему учителю,

с уважением и признательностью

Одним из естественных вопросов универсальной алгебры является вопрос возможности для объектов, определяемых на универсальных алгебрах тем или иным синтаксическим образом (уравнениями, формулами и т. п.), быть определимыми в терминах производных объектов этих алгебр: подалгебр, конгруэнций, авто-, эпиморфизмов и пр. В частности, это касается функций, определимых на универсальных алгебрах: термальных, условно термальных, неявных и т. д. Представляет в этом плане интерес вопрос о функциях, график которых является алгебраическим или кусочно алгебраическим множеством, имеется в виду параллель с понятием алгебраической функции в теории функций действительного переменного.

Прежде всего напомним, что в теории функций действительного переменного функция $f\left(x_{1}, \ldots, x_{n}\right)$ определяется как алгебраическая, если в некоторой окрестности любой точки из области ее определения она задается неявно с помощью некоторого алгебраического уравнения.

Рассматривая аналогичное определение для произвольных универсальных алгебр (поскольку отсутствует однозначно определенная каноническая топология на этих алгебрах и может не выполняться для них, в общем случае, условие нётеровости по уравнениям), естественно приходим к определению функиии $f\left(x_{1}, \ldots, x_{n}\right)$ как алгебрачческой для алгебры $\mathfrak{A}=\langle A ; \sigma\rangle$, если график этой функции $G r f=\left\{\left\langle a_{1}, \ldots, a_{n}, f\left(a_{1}, \ldots, a_{n}\right)\right\rangle \mid a_{i} \in A\right\}$ является объединением алгебраических для $\mathfrak{A}$ множеств. Напомним также, что множество $B \subseteq A^{m}$ называется алгебраическим для алгебры $\mathfrak{A}$, если $B$ есть совокупность решений в $\mathfrak{A}$ некоторой (возможно, бесконечной) системы термальных уравнений

$$
t_{i}^{1}\left(x_{1}, \ldots, x_{m}\right)=t_{i}^{2}\left(x_{1}, \ldots, x_{m}\right) \quad(i \in I)
$$

сигнатуры $\sigma$. Далее множества, являющиеся объединением алгебраических множеств, будем называть обобщенно алгебраическими.

(c) 2020 Пинус А. Г. 
Функиию $f\left(x_{1}, \ldots, x_{n}\right)$ назовем глобально алгебраической для алгебры $\mathfrak{A}$, если ее график является алгебраическим множеством этой алгебры.

Внутренним гомоморфизмом алгебры $\mathfrak{A}$ называется любой гомоморфизм между ее подалгебрами. Совокупность всех внутренних гомоморфизмов алгебры $\mathfrak{A}$ обозначим через $\operatorname{Ihm} \mathfrak{A}$. Функиию $f\left(x_{1}, \ldots, x_{n}\right)$ на алгебре $\mathfrak{A}$ определим как квазинеявную для $\mathfrak{A}$, если она коммутирует со всеми внутренними гомоморфизмами этой алгебры. Определение дано по аналогии с определением неявных функций на псевдомногообразиях универсальных алгебр от Эйленберга и Шутценберже [1], но в отличие от работы [2] где определены неявные для алгебры $\mathfrak{A}$ функции и дано их синтаксическое описание, не требуем при определении квазинеявной функции принадлежности элемента $f\left(a_{1}, \ldots, a_{n}\right)$ подалгебре алгебры $\mathfrak{A}$, порожденной элементами $a_{1}, \ldots, a_{n}$ из $\mathfrak{A}$.

В связи с этим необходимо уточнить, что, говоря о коммутируемости некоторой функции $f\left(x_{1}, \ldots, x_{n}\right)$, определенной на основном множестве $A$ алгебры $\mathfrak{A}$, с некоторым частичным отображением $h$ этого множества в себя, имеем в виду равенство $h f\left(a_{1}, \ldots, a_{n}\right)=f\left(h\left(a_{1}\right), \ldots, h\left(a_{n}\right)\right)$ для всех элементов $a_{1}, \ldots, a_{n}$ из области $\operatorname{Dom} h$ определения отображения $h$ таких, что $f\left(a_{1}, \ldots, a_{n}\right) \in \operatorname{Dom} h$, т. е. замкнутость относительно $h$ графика функции $f$.

Очевидно, что всякая алгебраическая, в том числе глобально алгебраическая, функция для алгебры $\mathfrak{A}$ квазинеявная для $\mathfrak{A}$. Покажем, что для алгебраических функций верно и обратное, в то время как для глобально алгебраических функций это не так.

Начнем с последнего, т. е. укажем универсальную алгебру $\mathfrak{A}=\langle A ; \sigma\rangle$ и некоторую квазинеявную для нее функцию $f$, не глобально алгебраическую для $\mathfrak{A}$.

Пусть $\mathfrak{A}=\langle A ; \sigma\rangle$, где сигнатура $\sigma$ состоит из одной унарной функции $g(x)$, а $A=A_{0} \cup A_{1}$ и $g$ является 2-циклом на $A_{0}=\left\{a_{0}^{0}, a_{0}^{1}\right\}$ и 3 -циклом на $A_{1}=\left\{a_{1}^{0}, a_{1}^{1}, a_{1}^{2}\right\}$. Очевидным образом внутренние гомоморфизмы алгебры $\mathfrak{A}$ суть внутренние гомоморфизмы ее подалгебр $\mathfrak{A}_{0}=\left\langle A_{0} ; \sigma\right\rangle$ и $\mathfrak{A}_{1}=\left\langle A_{1} ; \sigma\right\rangle$ либо теоретико-множественные объединения таких гомоморфизмов, т. е. имеют вид $\varphi_{0}, \varphi_{1}$, либо $\varphi_{0} \cup \varphi_{1}$, где $\varphi_{0}(x)=g^{i_{0}}(x)$ для $x \in A_{0}$ и $i_{0} \in\{0,1\}$ и $\varphi_{1}(x)=g^{i_{1}}(x)$ для $x \in A_{1}$ и $i_{1} \in\{0,1,2\}$. Функцию $f(x)$ на $A$ определим следующим обра3ом: $f(x)=g(x)$ на $A_{0}$ и $f(x)=g^{2}(x)$ на $A_{1}$. Очевидно, что она коммутирует со всеми внутренними гомоморфизмами алгебры $\mathfrak{A}$, т. е. квазинеявная для $\mathfrak{A}$. Заметим, что $f$ не глобально алгебраическая для $\mathfrak{A}$, т. е. ее график $\operatorname{Gr} f$ не является совокупностью решений в $\mathfrak{A}$ никакой системы термальных уравнений сигнатуры $\sigma$ от переменных $x_{1}, x_{2}$. Прежде всего, в силу определения алгебры $\mathfrak{A}$ любой $\sigma$-терм на $\mathfrak{A}$ эквивалентен одному из термов $g^{m}(x)$, где $m=0,1, \ldots, 5$ и $g^{0}(x)=x$. Таким образом, термальные уравнения от $x_{1}, x_{2}$ на алгебре $\mathfrak{A}$ имеют вид: либо

(1) $g^{m_{1}}\left(x_{i}\right)=g^{m_{2}}\left(x_{i}\right)$, где $i=1,2$ и $m_{1}, m_{2} \in\{0,1, \ldots, 5\}$, либо

(2) $g^{p_{1}}\left(x_{1}\right)=g^{p_{2}}\left(x_{2}\right)$, где $p_{1}, p_{2} \in\{0,1, \ldots, 5\}$.

Будем считать, что эти уравнения истинны на элементах $\left\langle x_{1}, x_{2}\right\rangle$ множества Gr $f$. Истинность уравнений вида (1) на элементах $a_{0}^{0}$ и $a_{1}^{0}$ возможна лишь в случае истинности сравнений $m_{0} \equiv m_{2}(\bmod 2)$ и $m_{1} \equiv m_{2}(\bmod 3)$, последнее возможно лишь в случае $m_{1}=m_{2}$, т. е. в случае тривиальности уравнений подобного вида. Истинность уравнения вида (2) на элементах $x_{1}=a_{0}^{0}$ и $x_{2}=f\left(a_{0}^{0}\right)=g\left(a_{0}^{0}\right)$ влечет истинность сравнения $p_{1}=p_{2}+1$ 
$(\bmod 2)$, а на элементах $x_{1}=a_{1}^{0}$ и $x_{2}=f\left(a_{1}^{0}\right)=g^{2}\left(a_{1}^{0}\right)$ - истинность сравнения $p_{1}=p_{2}+2(\bmod 3)$. Поскольку одновременная истинность этих сравнений для $p_{1}, p_{2} \in\{0,1, \ldots, 5\}$ невозможна, на элементах множества $\operatorname{Gr} f$ выполнимы лишь тривиальные уравнения и его алгебраическое замыкание есть $A^{2}$, т. е. множество $\operatorname{Gr} f$ не алгебраическое, а функция $f(x)$ не глобально алгебраическая для алгебры $\mathfrak{A}$.

Покажем, что понятия алгебраической и квазинеявной функций для универсальных алгебр равносильны. Это непосредственно вытекает из следующего утверждения.

Лемма 1. Множество $B \subseteq A^{m}$ обобщенно алгебраическое для алгебры $\mathfrak{A}=\langle A ; \sigma\rangle$ тогда и только тогда, когда оно замкнуто относительно внутренних гомоморфизмов этой алгебры (т. е. когда для любого кортежа $\left\langle a_{1}, \ldots, a_{m}\right\rangle \in B$ и любого $\varphi \in \operatorname{Ihm} \mathfrak{A}$ в случае, когда $a_{1}, \ldots, a_{m} \in \operatorname{dom} \varphi$, имеет место и включение $\left.\left\langle\varphi\left(a_{1}\right), \ldots, \varphi\left(a_{m}\right)\right\rangle \in B\right)$.

ДокАЗАтЕЛЬСтво. Если множество $B \subseteq A^{m}$ является объединением $\bigcup_{i \in I} B_{i}$ алгебраических для алгебры $\mathfrak{A}=\langle A ; \sigma\rangle$ множеств $B_{i}$, то, поскольку очевидным образом алгебраические множества $B_{i}$ замкнуты относительно внутренних гомоморфизмов алгебры $\mathfrak{A}$, таковым будет и множество $B$.

Обратно, пусть множество $B \subseteq A^{m}$ замкнуто относительно внутренних гомоморфизмов алгебры $\mathfrak{A}$. Пусть $\left\langle a_{1}, \ldots, a_{m}\right\rangle \in B$ и $D_{\left\langle a_{1}, \ldots, a_{m}\right\rangle}^{+}\left(x_{1}, \ldots, x_{m}\right)-$ позитивная диаграмма кортежа $\left\langle a_{1}, \ldots, a_{m}\right\rangle$ в алгебре $\mathfrak{A}$, т. е. совокупность всех термальных уравнений

$$
t_{j}^{1}\left(x_{1}, \ldots, x_{m}\right)=t_{j}^{2}\left(x_{1}, \ldots, x_{m}\right) \quad(j \in J),
$$

истинных на кортеже $\left\langle a_{1}, \ldots, a_{m}\right\rangle$ в алгебре $\mathfrak{A}$. Таким образом, для любого кортежа $\left\langle b_{1}, \ldots, b_{m}\right\rangle \in A^{m}$ истинность уравнений из $D_{\left\langle a_{1}, \ldots, a_{m}\right\rangle}^{+}\left(x_{1}, \ldots, x_{m}\right)$ на $\left\langle b_{1}, \ldots, b_{m}\right\rangle$ равносильна существованию внутреннего гомоморфизма $\varphi$ алгебры $\mathfrak{A}$ такого, что $\varphi\left(a_{i}\right)=b_{i}$ для $i=1, \ldots, m$. Тем самым алгебраическое для $\mathfrak{A}$ множество

$$
B_{\left\langle a_{1}, \ldots, a_{m}\right\rangle}=\left\{\left\langle b_{1}, \ldots, b_{m}\right\rangle \in A^{m} \mid \mathfrak{A} \models D_{\left\langle a_{1}, \ldots, a_{m}\right\rangle}^{+}\left(b_{1}, \ldots, b_{m}\right)\right\}
$$

является подмножеством множества $B$, а так как $\left\langle a_{1}, \ldots, a_{m}\right\rangle \in B_{\left\langle a_{1}, \ldots, a_{m}\right\rangle}$, то $B=\bigcup_{\left\langle a_{1}, \ldots, a_{m}\right\rangle \in B} B_{\left\langle a_{1}, \ldots, a_{m}\right\rangle}$, т. е. $B$ является обобщенно алгебраическим для алгебры $\mathfrak{A}$ множеством, и лемма доказана.

Непосредственно из утверждения этой леммы и отмеченного выше факта, что любая алгебраическая для алгебры $\mathfrak{A}$ функция квазинеявная для этой алгебры, вытекает

Теорема 1. Для любой универсальной алгебры $\mathfrak{A}$ функция $f$ алгебраическая для $\mathfrak{A}$ тогда и только тогда, когда она квазинеявная для $\mathfrak{A}$.

Напомним, что алгебра $\mathfrak{A}$ называется (см. [3]) эквациональной областью тогда и только тогда, когда любое объединение конечного числа ее алгебраических множеств является алгебраическим множеством для алгебры $\mathfrak{A}$. Эквациональными областями являются, в частности, любые дискриминаторные алгебры. Другие многочисленные примеры эквациональных областей см., к примеру, в [3].

Таким образом, для конечных эквациональных областей понятия алгебраической и глобально алгебраической функции совпадают, и из теоремы 1 непосредственно вытекает 
Следствие 1. Функция $f\left(x_{1}, \ldots, x_{n}\right)$ глобально алгебраична для конечной эквациональной области $\mathfrak{A}$ тогда и только тогда, когда $f$ квазинеявна для $\mathfrak{A}$.

Для любого кортежа $\left\langle b_{1}, \ldots, b_{m}\right\rangle$ элементов алгебры $\mathfrak{A}=\langle A ; \sigma\rangle$ кортеж ее элементов $\left\langle a_{1}, \ldots, a_{m}\right\rangle$ назовем Ihm $\mathfrak{A}$-образом кортежа $\left\langle b_{1}, \ldots, b_{m}\right\rangle$, если найдется $\varphi \in \operatorname{Ihm} \mathfrak{A}$, область определения которого включает в себя $\left\{b_{1}, \ldots, b_{m}\right\}$, и при этом $\varphi\left(b_{i}\right)=a_{i}$ для $i=1, \ldots, m$.

Пусть $\mathfrak{A}^{\prime}-$ некоторое расширение алгебры $\mathfrak{A}=\langle A ; \sigma\rangle$. Множество $B \subseteq$ $A^{m}$ назовем Ihm $\mathfrak{A}^{\prime}$-насыщенным в $\mathfrak{A}$ относительно кортежс $\left\langle b_{1}, \ldots, b_{m}\right\rangle$ элементов алгебры $\mathfrak{A}^{\prime}$, если кортежи из $B$ являются $\operatorname{Ihm} \mathfrak{A}^{\prime}$-образами кортежа $\left\langle b_{1}, \ldots, b_{m}\right\rangle$ и для любого $\operatorname{Ihm} \mathfrak{A}^{\prime}$-образа кортежа $\left\langle b_{1}, \ldots, b_{m}\right\rangle$, состоящего из элементов алгебры $\mathfrak{A}$, этот $\operatorname{Ihm} \mathfrak{A}^{\prime}$-образ входит в $B$, т. е. если $B=\left\{\left\langle a_{1}, \ldots, a_{m}\right\rangle \in\right.$ $A^{m} \mid\left\langle a_{1}, \ldots, a_{m}\right\rangle$ есть Ihm $\mathfrak{A}^{\prime}$-образ кортежа $\left.\left\langle b_{1}, \ldots, b_{m}\right\rangle\right\}$.

Лемма 2. Для любой универсальной алгебры $\mathfrak{A}=\langle A ; \sigma\rangle$ множество $B \subseteq$ $A^{m}$ алгебраическое для $\mathfrak{A}$ тогда и только тогда, когда для некоторого расширения $\mathfrak{A}^{\prime}$ алгебры $\mathfrak{A}$ и некоторого кортежа $\left\langle b_{1}, \ldots, b_{m}\right\rangle$ элементов из $\mathfrak{A}^{\prime}$ множество $B$ является Ihm $\mathfrak{A}^{\prime}$-насыщенным в $\mathfrak{A}$ относительно этого кортежа $\left\langle b_{1}, \ldots, b_{m}\right\rangle$.

ДоказАтельСтво. Пусть для алгебры $\mathfrak{A}=\langle A ; \sigma\rangle$ множество $B \subseteq A^{m}$ $\operatorname{Ihm} \mathfrak{A}^{\prime}$-насыщенно для некоторого расширения $\mathfrak{A}^{\prime}$ алгебры $\mathfrak{A}$ и некоторого кортежа $\left\langle b_{1}, \ldots, b_{m}\right\rangle$ элементов из $\mathfrak{A}^{\prime}$. Тогда из соответствующих определений непосредственно следует, что

$$
B=\left\{\left\langle a_{1}, \ldots, a_{m}\right\rangle \in A^{m} \mid \mathfrak{A} \models D_{\left\langle b_{1}, \ldots, b_{m}\right\rangle}^{+}\left(a_{1}, \ldots, a_{m}\right)\right\},
$$

т. е. $B$ - алгебраическое множество для алгебры $\mathfrak{A}$.

Покажем обратное. Пусть $B \subseteq A^{m}$ - некоторое алгебраическое для алгебры $\mathfrak{A}$ множество и $B=\left\{\left\langle b_{1}^{i}, \ldots, b_{m}^{i}\right\rangle \mid i \in I\right\}$. Пусть

$$
T\left(x_{1}, \ldots, x_{m}\right)=\bigcap_{i \in I} D_{\left\langle b_{1}^{i}, \ldots, b_{m}^{i}\right\rangle}^{+}\left(x_{1}, \ldots, x_{m}\right) .
$$

Алгебраичность множества $B$ означает, что

$$
B=\left\{\left\langle a_{1}, \ldots, a_{m}\right\rangle \in A^{m} \mid \mathfrak{A} \models T\left(a_{1}, \ldots, a_{m}\right)\right\} .
$$

В качестве искомого расширения $\mathfrak{A}^{\prime}$ алгебры $\mathfrak{A}$ подобно тому, как это делается в доказательстве теоремы 1 из [4], рассмотрим алгебру $\mathfrak{A}^{A^{m}}$ (отождествляя элементы $a$ алгебры $\mathfrak{A}$ с элементами $\bar{a}$ из $\mathfrak{A}^{A^{m}}$ такими, что $\bar{a}_{(j)}=a$ для любого $j \in A^{m}$ ). Элементы $b_{l} \in \mathfrak{A}^{A^{m}}$ (для $l=1, \ldots, m$ ) определим так, что $\left\{\left\langle b_{1}(j), \ldots, b_{m}(j)\right\rangle \mid j \in A^{m}\right\}=B$. Тогда $D_{\left\langle b_{1}, \ldots, b_{m}\right\rangle}^{+}\left(x_{1}, \ldots, x_{m}\right)=T\left(x_{1}, \ldots, x_{m}\right)$. В частности, $B$ состоит из Ihm $\mathfrak{A}^{\prime}$-образов кортежа $\left\langle b_{1}, \ldots, b_{m}\right\rangle$ в алгебре $\mathfrak{A}$, а равенство $(* *)$ означает $\operatorname{Ihm} \mathfrak{A}^{\prime}$-насыщенность множества $B$ в алгебре $\mathfrak{A}$ относительно кортежа $\left\langle b_{1}, \ldots, b_{m}\right\rangle$. Лемма доказана.

Обозначим совокупности алгебраических, глобально алгебраических и квазинеявных функций для алгебры $\mathfrak{A}=\langle A ; \sigma\rangle$ через Algf $\mathfrak{A}, \operatorname{GAlgf} \mathfrak{A}$ и $\operatorname{QImpf} \mathfrak{A}$ соответственно. Через FunA обозначим совокупность всех функций на $A$. Имеют место следующие очевидные включения между этими совокупностями для любой алгебры $\mathfrak{A}:$ GAlgf $\mathfrak{A} \subseteq \operatorname{Algf} \mathfrak{A}=\operatorname{Qimpf} \mathfrak{A} \subseteq$ FunA .

Традиционный интерес представляет вопрос описания алгебр $\mathfrak{A}$, для которых перечисленные совокупности совпадают между собой.

Алгебру $\mathfrak{A}=\langle A ; \sigma\rangle$ назовем алгебраически примальной (аналог определения примальности для конечных алгебр с заменой клона термальных функций 
алгебры $\mathfrak{A}$ совокупностью $\operatorname{Algf} \mathfrak{A})$, если имеет место равенство Algf $\mathfrak{A}=$ FunA . Алгебру $\mathfrak{A}$ назовем абсолютно жесткой, если все ее внутренние гомоморфизмы тождественны.

Следствие 2. Алгебра $\mathfrak{A}$ алгебраически примальна тогда и только тогда, когда она абсолютно жестка.

Действительно, пусть $\mathfrak{A}=\langle A ; \sigma\rangle$ - алгебраически примальная алгебра и $\varphi-$ некоторый нетождественный внутренний гомоморфизм алгебры $\mathfrak{A}$, т. е. $\varphi(a)=b \neq a$ для некоторого $a \in A$. Но тогда алгебраическая по предположению константная на $A$ функция $g(x)=a$ не коммутирует с $\varphi$. Это противоречие и доказывает тривиальности всех внутренних гомоморфизмов алгебры A. Верно и обратное: если все внутренние гомоморфизмы алгебры $\mathfrak{A}=\langle A ; \sigma\rangle$ тривиальны, то все функции на множестве $A$ квазинеявны для $\mathfrak{A}$, а значит, в силу теоремы 1 они являются алгебраическими для алгебры $\mathfrak{A}$.

Также непосредственно из утверждения леммы 2 вытекает

Теорема 2. Для любой универсальной алгебры $\mathfrak{A}$ следующие условия равносильны:

(1) имеет место равенство GAlgf $\mathfrak{A}=\operatorname{Algf} \mathfrak{A}$,

(2) для любой алгебраической для алгебры $\mathfrak{A}$ функции $f\left(x_{1}, \ldots, x_{n}\right)$ ее график является Ihm $\mathfrak{A}^{\prime}$-насыщенным в алгебре $\mathfrak{A}$ множеством относительно некоторого кортежа элементов из некоторого расширения $\mathfrak{A}^{\prime}$ алгебры $\mathfrak{A}$.

Благодарность. Автор признателен рецензенту, указавшему на необходимость более тщательного, чем в первоначальном варианте статьи, определения ситуации коммутирования функции с частичным отображением и на то, что совокупность алгебраических, глобально алгебраических и квазинеявных функций на алгебре не обязаны быть клонами, а также за другие его замечания.

\section{ЛИТЕРАТУРА}

1. Eilenberg S., Schutzenberger M. P. On pseudovarieties // Adv. Math. 1979. V. 19, N 3. P. 413-418.

2. Пинус А. Г. Неявно эквивалентные универсальные алгебры // Сиб. мат. журн. 2012. T. 53, № 5. С. 1077-1090.

3. Даниярова Э. Ю., Мясников А. Г., Ремесленников В. Н. Алгебраическая геометрия над алгебраическими системами. Новосибирск: Изд-во СО РАН, 2016.

4. Пинус А. Г. n-Алгебраически полные алгебры, псевдопрямые произведения и оператор алгебраического замыкания на подмножествах универсальных алгебр // Сиб. журн. чистой и прикл. математики. 2016. Т. 16, № 4. С. 97-102.

Поступила в редакцию 25 мая 2019 г.

После доработки 5 октлбря 2019 г.

Принята к публикации 8 апреля 2020 г.

Пинус Александр Георгиевич

Новосибирский гос. технический университет, пр. К. Маркса, 20, Новосибирск 630092

ag.pinus@gmail.com 